\title{
Like sign top quark pair production at linear colliders
}

\author{
Wei-Shu Hou ${ }^{a}$, Guey-Lin Lin ${ }^{b}$ \\ a Department of Physics, National Taiwan University, Taipei, Taiwan 10764, ROC \\ ${ }^{\mathrm{b}}$ Institute of Physics, National Chiao Tung University, Hsinchu, Taiwan 30050, ROC \\ Received 28 January 1996 \\ Editor: H. Georgi
}

\begin{abstract}
In the general two Higgs doublet model with flavor changing neutral Higgs couplings, neutral Higgs bosons may decay dominantly via $t \bar{c}$ or $\bar{t} c$ final states. At the linear collider, $e^{+} e^{-} \rightarrow h^{0} A^{0}$ or $H^{0} A^{0}$ production processes may result in $b \bar{b} t \bar{c}$, $W^{+} W^{-} t \bar{c}$ or $t t \bar{c} \bar{c}$ (or $\bar{t} \bar{t} c c$ ) final states. The process $\gamma \gamma \rightarrow h^{0}, A^{0} \rightarrow t \bar{c}$ is also promising, but $e^{+} e^{-} \rightarrow\left(h^{0}, H^{0}\right) Z^{0} \rightarrow t \bar{c} Z^{0}$ is relatively suppressed. The possibility of observing like sign lepton pairs, usually the hallmark for neutral meson mixing, is quite interesting since $T^{0}$ mesons do not even form.
\end{abstract}

PACS: $14.80 . \mathrm{Dq} ; 14.80 . \mathrm{Gt} ; 12.15 . \mathrm{Cc} ; 13.90 .+\mathrm{i}$

Like sign dilepton pair production is the hallmark for heavy neutral meson-antimeson mixing. The standard model (SM) predicts rather small mixing effects for mesons containing $u$-type quarks. Furthermore, due to its heaviness, the top quark decays before the $T_{u}^{0}$ or $T_{c}^{0}$ mesons could form. Thus, unlike the $b$ quark case, we do not expect same sign dileptons from $t \bar{t}$ pair production. Effects beyond the standard model are not expected to change this, since the Tevatron data $\lfloor 1\rfloor$ is in good agreement with $t \rightarrow b W$ decay dominance expected in SM. In this note we report [2] the intriguing possibility of producing like sign top quark pairs at linear $e^{+} e^{-}$colliders, within the context of a general two Higgs doublet model (2HDM) that possesses flavor changing neutral Higgs ( $\mathrm{FCNH}$ ) couplings [3-5].

Atwood, Reina and Soni have recently studied [6] FCNH loop induced $e^{+} e^{-} \rightarrow \gamma^{*}, Z^{*} \rightarrow t \bar{c}$ transitions at linear colliders and find a rather small rate. They also propose [7] to study the tree level $s$-channel
FCNH process $\mu^{+} \mu^{-} \rightarrow$ neutral scalars $\rightarrow t \bar{c}$. Here, we explore FCNH coupling effects in Higgs boson production processes at a $500 \mathrm{GeV} e^{+} e^{-}$Next Linear Collider (NLC). We find it to be promising, both for singlc top $t \bar{c}+X$, as wcll as for the more intriguing like sign top pair $t t \bar{c} \bar{c}$ final states.

Let us briefly review the model under consideration. With two Higgs doublets $\Phi_{1}$ and $\Phi_{2}$, in general one has FCNH couplings. Because of stringent bounds from $\mu \rightarrow e \gamma$ decay, $K^{0}-\bar{K}^{0}$ and $B^{0}-\bar{B}^{0}$ mixings, etc., it is customary [8] to strictly enforce the absence of FCNH couplings at tree level. This is readily achieved via some discrete symmetry that allows just one source of mass for each given fermion charge [9], much like in SM. However, inspired by the quark mass and mixing hierarchy pattern

$$
\begin{aligned}
& m_{1} \ll m_{2} \ll m_{3}, \\
& \left|V_{u b}\right|^{2} \ll\left|V_{c b}\right|^{2} \ll\left|V_{u s}\right|^{2} \ll 1,
\end{aligned}
$$

that emerged since the early 1980 's, Cheng and Sher 
[3] suggested that low energy flavor changing neutral currents could be naturally suppressed, without the need to invoke discrete symmetries. Let us elaborate on this observation.

We shall assume $C P$ invariance throughout the paper, leaving out even the possibility of spontaneous $C P$ violation [10]. Since both $\Phi_{1}$ and $\Phi_{2}$ develop real vacuum expectation values (v.e.v.), one can redefine the fields and choose one doublet as the "mass giver", i.e. $\left\langle\phi_{1}^{0}\right\rangle=v / \sqrt{2},\left\langle\phi_{2}^{0}\right\rangle=0$, where $v \simeq 246$ $\mathrm{GeV}$. The usual $2 \mathrm{HDM}$ parameter $\tan \beta \equiv v_{1} / v_{2}$ gets rotated away by the freedom to make linear redefinitions. One readily sees that $[5] \sqrt{2} \operatorname{Re} \phi_{1}^{0}$ has diagonal couplings. It is, however, not a mass eigenstate. For $\Phi_{2}$ related fields, we have,

$$
\begin{aligned}
& \left(\bar{u}_{L} \xi^{(u)} u_{R}+\bar{d}_{L} \xi^{(d)} d_{R}\right) \sqrt{2} \operatorname{Re} \phi_{2}^{0} \\
& \quad+\left(-\bar{u}_{L} \xi^{(u)} u_{R}+\bar{d}_{L} \xi^{(d)} d_{R}\right) i \sqrt{2} \operatorname{Im} \phi_{2}^{0} \\
& \quad-\bar{d}_{L} V^{\dagger} \xi^{(u)} u_{R} \sqrt{2} \phi_{2}^{-}+\bar{u}_{L} V \xi^{(d)} d_{R} \sqrt{2} \phi_{2}^{+} \\
& \quad+\text { H.c. }
\end{aligned}
$$

where $\xi^{(u, d)}$ are in general not diagonal, but $V^{(\dagger)} \xi \simeq$ $\xi$, since the KM matrix $V \simeq 1$.

At first sight, the Yukawa coupling matrices $\xi^{(i, d)}$ may appear to be completely general. However, in some arbitrary basis where $\left\langle\phi_{1}^{0}\right\rangle=v_{1} / \sqrt{2},\left\langle\boldsymbol{\phi}_{2}^{0}\right\rangle=$ $v_{2} / \sqrt{2}$, quark mass matrices consists of two parts, $m=m^{(1)}+m^{(2)}$. To sustain Eq. (1), unless finetuned cancellations are implemented, one would expect that the off diagonal elements of $m^{(1)}$ and $m^{(2)}$, just like $m$ itself, should trickle off as one moves offdiagonal. The rotation (linear redefinition) by angle $\beta=\tan ^{-1}\left(v_{1} / v_{2}\right)$ to Eq. (2) should not change this property. Hence, data (Eq. (1)) suggest that $\xi$ cannot be arbitrary. In this vein, Cheng and Sher proposed [3] the ansatz

$\xi_{i j} \sim \sqrt{m_{i} m_{j}} / v$.

The bonus was that FCNH couplings involving lower generation fermions are naturally suppressed, without the need to push FCNH Higgs boson masses to way beyond the v.e.v. scale [11]. Inspecting Eq. (1) again, a weaker ansatz is possible [2],

$\xi_{i j}=\mathcal{O}\left(V_{i 3} V_{j 3}\right) m_{3} / v$.

According to the mass-mixing pattern, the ChengSher ansatz of Eq. (3) corresponds to $\xi_{i j}=$
$\mathcal{O}\left(V_{i j} V_{j 3}\right) m_{3} / v$. Note that in both cases, $\xi_{c t}$ is the largest possible FCNH coupling, and the associated phenomenology is the most interesting $[4,12]$. We shall assume that Eq. (3) holds with equality sign in our following discussions. Our numerical results can be easily scaled if equality does not hold.

The pseudoscalar $A^{0} \equiv \sqrt{2} \operatorname{Im} \phi_{2}^{0}$ and charged scalar $H^{ \pm} \equiv \phi_{2}^{ \pm}$are already physical Higgs bosons, but the neutral $C P$ even Higgs bosons $H^{0}$ and $h^{0}$ are mixtures of $\sqrt{2} \operatorname{Re} \phi_{1}^{0}$ and $\sqrt{2} \operatorname{Re} \phi_{2}^{0}$. The mixing angle $\sin \alpha$, a physical parameter, is determined by the Higgs potential. In the limit of $\sin \alpha \rightarrow 0$, often assumed by various authors $[5-7], H^{0} \leadsto \sqrt{2} \operatorname{Re} \phi_{1}^{0}$ becomes the "standard" Higgs boson with diagonal couplings, while $h^{0} \rightsquigarrow \sqrt{2} \operatorname{Re} \phi_{2}^{0}$ has Yukawa couplings as in Eqs. (3) or (4), but decouples from vector bosons or charged Higgs bosons, just like $A^{0}$. Our convention for $H^{0}$ and $h^{0}$ differs from the minimal supersymmetric SM (MSSM) [8], where $h^{0}$ is taken as the lighter $C P$ even neutral scalar.

Constraints on the general 2HDM has been studied by various authors. For $K^{0}$ and $B^{0}$ mixings, one finds [3,13] a rather weak bound of $m_{h^{0}} \gtrsim 80 \mathrm{GeV}$, with a more stringent bound for $A^{0}$. These bounds could weaken, for example, if one uses Eq. (4) instead of Eq. (3). For $\mu \rightarrow e \gamma$, an interesting two loop effect dominates over one loop diagrams [14]. From Fig. 4 of Ref. [14], with $m_{t} \simeq 175 \mathrm{GeV}$, one finds a bound of $m_{h^{0}} \gtrsim 150 \mathrm{GeV}$. The bound for $A^{0}$ is weaker since it does not couple to vector bosons and unphysical scalar bosons. If $\sin \alpha \rightarrow 0$, the $h^{0}$ bound would also weaken. A third, less direct constraint on FCNH Higgs boson masses is from the recent experimental observation of inclusive $b \rightarrow s \gamma$ decays. Within the the so-called Model II of 2HDM [8] (automatically realized in MSSM), the CLEO Collaboration gives [15] a bound of $m_{H^{+}}>250 \mathrm{GeV}$. This bound should weaken for our case because of the freedom in $\xi^{(u, d)}$ as compared to Model II. Inclusion of next-to-leading order QCD corrections also tends to soften the bound [16]. Thus, we take $m_{H^{+}} \gtrsim 150-250 \mathrm{GeV}$ as a reasonable lower bound, which is rather consistent with the bounds on FCNH neutral scalar bosons. The upshot of our discussion on low energy constraints is that,

$v \sim m$ (FCNH Higgs) $\gtrsim m_{t}$

is not only reasonable, but quite likely. Although $t \rightarrow$ 


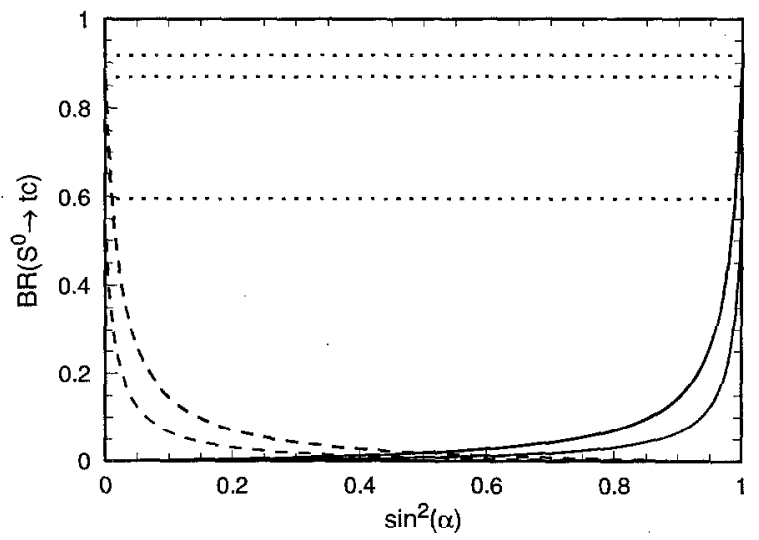

Fig. 1. BR $\left(S^{0} \rightarrow t \bar{c}+\bar{t} c\right)$ vs. $\sin ^{2} \alpha$ for $S=h$ (dash), $A$ (dot-dash) and $H$ (solid) and $m_{S^{0}}=200,250,300 \mathrm{GeV}$ (from bottom to top).

$c+$ scalar transitions $[4,12]$ are not excluded, we are more interested in Higgs bosons decaying into $t \bar{c}[4]$.

We will focus mainly on the mass domain of

$$
200 \mathrm{GeV}<m_{h^{0}, A^{0}}<2 m_{t} \simeq 350 \mathrm{GeV} .
$$

We plot in Fig. $1 \mathrm{BR}\left(S^{0} \rightarrow t \bar{c}+\bar{t} c\right)$ vs. $\sin ^{2} \alpha$, for $S=h, A$ and $H$ and $m_{S^{0}}=200,250,300 \mathrm{GeV}, A^{0}$ can decay only via $t \bar{c}$ and $f \bar{f}$ modes, and can be treated as independent of $\sin ^{2} \alpha$. Once $m_{A^{0}}$ is suitably above $t c$ threshold, the $t \bar{c}$ or $\bar{t} c$ modes dominate. The behavior for $h^{0}$ at $\sin \alpha=0$ is similar to $A^{0}$. However, as $\sin ^{2} \alpha$ grows, the $h^{0} \rightarrow V \bar{V}(V=W, Z)$ partial width grows rapidly, and $t \bar{c}$ branching ratio becomes rather suppressed. The case for $H^{0}$ is the same as $h^{0}$ under the interchange of $\sin ^{2} \alpha \longleftrightarrow \cos ^{2} \alpha$. The proximity of the $m_{h^{0}, H^{0}}=250$ and $300 \mathrm{GeV}$ curves is accidental.

One might think that the most promising channel for studying FCNH Higgs bosons is via the associated production process of $e^{+} e^{-} \rightarrow Z^{*} \rightarrow H^{0} Z^{0}$ and $h^{0} Z^{0}$ ( The $W^{-\downarrow} W^{--}$fusion process $e^{1} e^{-} \rightarrow \nu \bar{\nu}+H^{0}$ is subdominant for the range of Eq. (6)). This turns out to be not the case. We plot in Fig. 2 the cross section times branching ratio for the signature $e^{+} e^{-} \rightarrow$ $S^{0} Z^{0} \rightarrow t \bar{c} Z^{0}$ vs. $\sin ^{2} \alpha$, where $S=H, h$, again for $m_{S^{0}}=200,250,300 \mathrm{GeV}$. For $H^{0}$ this behaves as $x(1-x) /(A(1-x)+a x+\delta)$ where $x \equiv \sin ^{2} \alpha$, $A$ is related to the $H \rightarrow V \bar{V}$ ratc in SM, and $a, \delta$ are related to the $t \bar{c}$ and $b \bar{b}$ rate. For $h^{0}$ case one just interchanges $x \rightarrow 1-x$. Clearly, in the limit of $\sin \alpha=$ $0, H^{0}$ couples only flavor diagonally, while $h^{0}$ has no

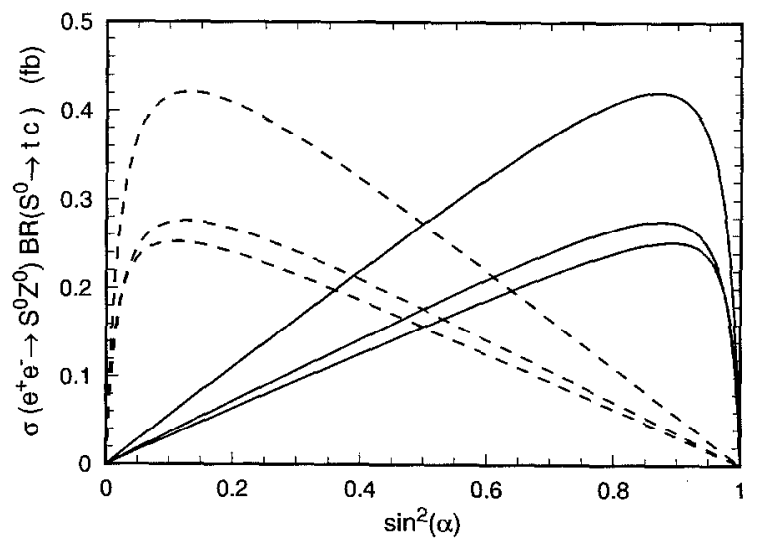

Fig. 2. $\sigma\left(e^{+} e^{-} \rightarrow S^{0} Z^{0}\right) \times \operatorname{BR}\left(S^{0} \rightarrow t \bar{c}\right)$ vs. $\sin ^{2} \alpha$, for $S=h$ (dash), $H$ (solid) and $m_{S^{0}}=200$ (lower curve), 250 (upper curve), 300 (middle curve) GeV.

production cross section. As $\sin \alpha$ grows, because of the mismatch in the production and decay process, the effective cross section for $t \bar{c}+Z^{0}$ associated production remains rather small, with the maximum of $0.43 \mathrm{fb}$ at $m_{h, H} \cong 237 \mathrm{GeV}$ and $\sin ^{2} \alpha\left(1-\sin ^{2} \alpha\right.$ for $\left.H^{0}\right) \cong 0.129$. This is but a fraction of the total $e^{+} e^{-} \rightarrow S^{0} Z^{0}$ cross section, which would not be easy to observe once one folds in various branching ratios for $t$ or $Z$ decay.

What is more promising is the $e^{+} e^{-} \rightarrow Z^{*} \rightarrow S^{0} A^{0}$ associated production process, where $S=h, H$. In the $\sin \alpha \rightarrow 0$ limit, one has $e^{+} e^{-} \rightarrow h^{0} A^{0}$ only, with cross section similar to the $H^{0} Z^{0}$ mode when phase space is similar. Since in this limit, $h^{0} \nrightarrow V \bar{V}$, the $t \bar{c}$ modc has a good chance to be the dominant final state for both $h^{0}$ and $A^{0}$. We immediately see the possibility of our purported $t t \bar{c} \bar{c}$ or $\bar{t} \bar{t} c c$ final states!

Let us proceed a bit more systematically. Taking $m_{A^{0}}>m_{h^{0}}$ for illustration (since $A^{0} \rightarrow t \bar{c}$ is quite likely to be dominant), we allow $m_{h^{0}}$ to be as low as $100 \mathrm{GeV}$, with $m_{A^{0}}$ in the range of Eq. (6), but $A^{0} \rightarrow h^{0} Z^{0}$ is kinematically suppressed or forbidden. For numerical illustration, we give in Table 1 the number of $h^{0} A^{0}$ events at an NLC with $50 \mathrm{fb}^{-1}$ integrated luminosity, for $\sin \alpha=0, m_{A}^{0}=200,250$ $\mathrm{GeV}, m_{h^{0}}=100,150,200 \mathrm{GeV}$, with the condition that $m_{A^{0}}-m_{h^{0}}<100 \mathrm{GeV}$. We see thal, up to phase space, a few $\times 10^{2}$ to $10^{3}$ raw events are expected. To determine the number of signal events, one could easily fold in branching ratios from Fig. 1, and $\cos ^{2} \alpha$ or 
Table 1

Number of $e^{+} e^{-} \rightarrow h^{0} A^{0}$ events for $\sin \alpha=0$ at $500 \mathrm{GeV} \mathrm{NLC}$ with $\int \mathcal{L} d t=50 \mathrm{fb}^{-1}$

\begin{tabular}{lll}
\hline$m_{A^{0}}(\mathrm{GeV})$ & $m_{h^{0}}(\mathrm{GeV})$ & $\mathcal{N}\left(h^{0} A^{0}\right)$ \\
\hline 200 & 100 & 1160 \\
200 & 150 & 900 \\
250 & 150 & 490 \\
200 & 200 & 520 \\
230 & 230 & $? ? ?$ \\
250 & 200 & 200 \\
250 & 250 & - \\
300 & 200 & - \\
\hline
\end{tabular}

$\sin ^{2} \alpha$ factors for the production cross section. These should then be compared with potential backgrounds, the chief ones being $e^{+} e^{-} \rightarrow t \bar{t}, W^{+} W^{-}, Z^{0} Z^{0}$ events, which are of order $3 \times 10^{4}, 4 \times 10^{5}, 3 \times 10^{4}$, respectively. Assuming $A^{0} \rightarrow t \bar{c}+\bar{t} c$ is predominant, three modes are of interest: $h^{0} \rightarrow b \bar{b}$, $t \bar{c}$ and $V \bar{V}$.

For $m_{h^{0}}<2 M_{W}$, or when $\sin ^{2} \alpha \ll 0.1$ but the $t \bar{c}$ mode is suppressed or forbidden, $h^{0} \rightarrow b \bar{b}$ would be the dominant decay mode. We have $\sigma\left(e^{+} e^{-} \rightarrow\right.$ $\left.h^{0} A^{0}\right) \times \operatorname{BR}\left(h^{0} \rightarrow b \bar{b}\right) \mathrm{BR}\left(A^{0} \rightarrow t \bar{c}+\bar{t} c\right) \sim 10$ $20 \mathrm{fb}$, which is quite sizable compared to the $t \bar{c} Z^{0}$ case. However, the $b \bar{b} t \bar{c}$ final state may take some effort to identify, since there is just one top quark. Although kinematic tricks could be played, but faced with backgrounds that are orders of magnitude higher, one would need very good $b$-tagging efficiency, and would likely need to know $m_{h^{0}}$ beforehand. However, the latter may have to be studied at the NLC itself, unless the intermediate mass Higgs search program (via $H \rightarrow \gamma \gamma$ detection) at the LHC turns out to be very successful. In any case a detailed Monte Carlo study would be necessary to determine whether this mode can be fruitfully studied.

For $\sin ^{2} \alpha \ll 0.1$, as can be seen from Fig. $1, h \rightarrow$ $t \bar{c}$ is likely dominant in the mass range of Eq. (6). We find $\sigma\left(e^{+} e^{-} \rightarrow h^{0} A^{0}\right) \times \mathrm{BR}\left(h^{0} \rightarrow t \bar{c}+\bar{t} c\right) \mathrm{BR}\left(A^{0} \rightarrow\right.$ $t \bar{c}+\bar{t} c) \sim 5 \mathrm{fb}$, which is slightly smaller than the previous case because of phase space. Clearly, $50 \%$ of this cross section goes into $t t \bar{c} \bar{c}$ or $\bar{t} \bar{t} c c$ final states, which is again larger than the $t \bar{c} Z^{0}$ case. We expect typically of order 130 such events. Folding in the semileptonic branching ratio, one expects $\sim 6$ events in the signal of
Table 2

Number of $e^{+} e^{-} \rightarrow h^{0} A^{0}$ events for $\sin \alpha=0$ at $600 \mathrm{GeV}$ NLC with $\int \mathcal{L} d t=50 \mathrm{fb}^{-1}$

\begin{tabular}{llc}
\hline$m_{A^{0}}(\mathrm{GeV})$ & $m_{h^{0}}(\mathrm{GeV})$ & $\mathcal{N}\left(h^{0} A^{0}\right)$ \\
\hline 200 & 100 & 1090 \\
200 & 150 & 880 \\
250 & 150 & 650 \\
200 & 200 & 720 \\
230 & 230 & 440 \\
250 & 200 & 500 \\
250 & 250 & 290 \\
300 & 200 & 280 \\
\hline
\end{tabular}

$e^{+} e^{-} \longrightarrow \ell^{ \pm} \ell^{\prime \pm}+\nu \nu+4 j$

where the 4 jets have flavor $b b \bar{c} \bar{c}$ or $\bar{b} \bar{b} c c$. While the number is a bit small, thanks to the large top quark mass, this distinctive signature has seemingly no background. In contrast, if one allows only one top to decay semileptonically, or if one tries to probe the equivalent number of $t \bar{t} c \bar{c}$ events, the single $\ell+\nu+6 j$ or opposite sign dilepton $\ell^{ \pm} \ell^{\prime \mp}+\nu \nu+4 j$ signatures would be swamped by $t \bar{t}$ or $W^{+} W^{-}$production background, which are orders of magnitude higher. In particular, standard $e^{+} e^{-} \rightarrow t \bar{t}$ pair production with hard gluon radiation may be especially irremovable. Since the effect demands $m_{h^{0}}+m_{A^{0}}>400 \mathrm{GeV}$, one is phase space limited at a $500 \mathrm{GeV} \mathrm{NLC.} \mathrm{If} \mathrm{the} \mathrm{center} \mathrm{of} \mathrm{mass} \mathrm{en-}$ ergy of the NLC could be increased to $600 \mathrm{GeV}$ or so, possible phase space or branching ratio suppressions for producing $e^{+} e^{-} \rightarrow h^{0} A^{0} \rightarrow t t \bar{c} \bar{c}+\bar{t} \bar{t} c c$ could be relieved. We illustrate this in Table 2, where one sees that higher mass Higgs boson pairs have larger cross section than in Table II. Taking into account from Fig. 1 that $t \bar{c}$ branching ratios approach 1 for Higgs mass heavier than $230 \mathrm{GeV}$, we see that an NLC with 600 GeV CM energy has much better chance of observing like sign top pair events than a $500 \mathrm{GeV}$ machine, assuming similar luminosities.

For $\sin ^{2} \alpha \gtrsim 0.1$ and $m_{h^{0}}>2 M_{W}, h^{0} \rightarrow W^{+} W^{-}$ and $Z^{0} Z^{0}$ decays are (pre) dominant, with $h^{0} \rightarrow t \bar{c}$ no more than $10 \%$, and like sign top quark pair final states become no longer visible. The case remains interesting, however, since $W^{+} W^{-} t \bar{c}$ or $Z^{0} Z^{0} t \bar{c}$ final states at the 5-10 fb level (taking into account both $h^{0}$ and $H^{0}$ ) are still quite conspicuous. One effectively has $W^{+} W^{-} W^{+} b \bar{c}$ in final state, which again has little background. In particular, one could still have like 
sign dilepton pairs as in Eq. (7). With $m_{h^{0}}$ or $m_{H^{0}}$ known from the LHC, this decay mode could be studied at the NLC in complete detail.

It is also possible to produce Higgs boson via the $\gamma \gamma \rightarrow S^{0}$ process [8]. We note that $A^{0}$ only couples to fermions, hence its effective coupling to photons is smaller than the SM Higgs boson. For $h^{0}$, if $\sin \alpha$ is very small, the case is again similar. As $\sin \alpha$ grows, the effective coupling would quickly become dominated by vector bosons and the production cross section could be larger. This is, however, offset by the reduction in $h^{0} \rightarrow t \bar{c}$ branching ratio. Since $\gamma \gamma \rightarrow S^{0} \rightarrow$ $t \bar{c}$ should have little background (e.g. $t \bar{c} \rightarrow W^{+} b \bar{c}$ can be distinguished from $W^{+} W^{-}$via $b$-tagging since $\left.\operatorname{BR}\left(W^{-} \rightarrow b \bar{q}\right)<10^{-3}\right)$, the number of events expected is [17]

$$
\begin{aligned}
& N\left(\gamma \gamma \rightarrow S^{0} \rightarrow t \bar{c}+\bar{t} c\right) \\
& \quad=4 \pi^{2} \Gamma\left(S^{0} \rightarrow \gamma \gamma\right) \operatorname{BR}\left(S^{0} \rightarrow t \bar{c}+\bar{t} c\right) / m_{S^{0}}^{2} \\
& \quad \times\left.\left(1+\left\langle\lambda \lambda^{\prime}\right\rangle\right)\left\langle d \mathcal{L}_{\gamma \gamma} / d m_{\gamma \gamma}\right\rangle\right|_{m_{\gamma \gamma}=m_{S^{0}} .}
\end{aligned}
$$

It is possible [18] to tune photon polarizations to have $\left\langle\lambda \lambda^{\prime}\right\rangle \sim+1$ and effective luminosities close to the the $e^{+} e^{-}$mode (i.e. $\sim 50 \mathrm{fb}^{-1}$ ). If such is the case, then one expects $10^{2}-10^{3}$ raw events, which should make $t \bar{c}$ detection possible if the branching ratio is not too suppressed. Note that the corresponding number of $\gamma \gamma \rightarrow W^{+} W^{-}$pairs is at the $10^{4}-10^{5}$ level.

We now compare our results with that of Atwood, Reina and Soni. For $e^{+} e^{--} \rightarrow \gamma^{*}, Z^{*} \rightarrow t \bar{c}$ via FCNH loop effects, they find $R^{t c} \equiv \sigma\left(e^{+} e^{-} \rightarrow t \bar{c}+\right.$ $\left.\bar{t}_{c}\right) / \sigma\left(e^{+} e^{-} \rightarrow \gamma^{*} \rightarrow \mu^{+} \mu^{-}\right) \lesssim$ few $\times 10^{-5}[6]$, which amounts to less than 0.1 event for a $500 \mathrm{GeV}$ NLC with $50 \mathrm{fb}^{-1}$ integrated luminosity. In case $h^{0}$ and $A^{0}$ are heavier than the range of Eq. (6), the loop induced cross section also goes down by another order of magnitude [6]. Thus, this process is unlikely to be observable at the NLC. Although phase space favored, loop suppression in this case is too severe. For $\mu^{+} \mu^{-} \rightarrow h^{0}, A^{0} \rightarrow t \bar{c}+\bar{t} c$, the process occurs at tree level and has a sizable cross section [7]. But in the limit of $\sin \alpha \rightarrow 0, h^{0}$ would also not decay via the $V \bar{V}$ mode, just like $A^{0}$. A rather fine-stepped energy scan would then be needed because of the narrowness of the $h^{0}$ and $A^{0}$ width. Together with the technological uncertainty for a high energy, high luminosity $\mu^{+} \mu^{-}$ collider [19], this process might be less straightfor- ward to study than at the NLC, including the $\gamma \gamma$ collider option via $\gamma \gamma \rightarrow h^{0}, A^{0}$.

As stated in the Introduction, the signature of like sign top pair production is rather analogous to observing $T^{0} T^{0}$ or $T^{0} T^{0}$ pairs via $T^{0}-T^{0}$ mixing. With top mesons not even forming, however, it is the associated production of $h^{0} A^{0}$ pairs, which each subsequently decay equally into $t \bar{c}$ or $\bar{t} c$ final states, that circumvents the usual condition of associated production of $t \bar{t}$ (or, $W^{+} W^{-}$) pairs in most processes. Since $h^{0}$ and $A^{0}$ contribute to $B-\bar{B}$ mixing, in a sense the like sign top pair production effect is related to neutral meson mixing phenomena. We know of no other way to make $t t$ or $\bar{t}$ pairs in an $e^{t} e^{-}$collider environment.

In summary, within a general two Higgs doublet model with FCNH couplings, $h^{0}, A^{0} \rightarrow t \bar{c}+\bar{t} c$ could be the dominant decay mode. The most intriguing consequence is the possibility of detecting like sign top pair production via $e^{+} e^{-} \rightarrow h^{0} A^{0} \rightarrow t t \bar{c} \bar{c}$ or $\bar{t} \bar{t} c c$, while single top $t \bar{c} b \bar{b}$ or $t \bar{c} W^{+} W^{-}$production are also detectable. In contrast, the $e^{+} e^{-} \rightarrow\left(H^{0}, Z^{0}\right) \rightarrow$ $t \bar{c} Z^{0}$ process is rather suppressed and not competitive. The number of events, hence the FCNH Higgs boson mass reach, could be extended if the collider energy is higher. It is also possible to study single FCNH Higgs production via $\gamma \gamma \rightarrow h^{0}, A^{0} \ldots+t \bar{c}+\bar{t} c$. Since the neutral Higgs bosons of minimal supersymmetric standard model couple to fermions in a flavor diagonal way, the observation of FCNH signals would rule out MSSM. We urge experimental colleagues to study signal vs. background issues carefully.

The work of W.S.H. is supported in part by grant NSC 85-2112-M-002-011, and G.L.L. by grant NSC 85-2212-M-009-006 of the Republic of China.

\section{References}

[1] F. Abe et al. (CDF Collaboration), Phys. Rev. Letl. 74 (1995) 2626;

S. Abachi et al. (D $\emptyset$ Collaboration), Phys. Rev. Lett. 74 (1995) 2632.

[2] Preliminary results were reported at the Third Linear $e^{+} e^{-}$ Collider Workshop (LCWS95), September 1995, MoriokaAppi, Japan.

[3] T.P. Cheng and M. Sher, Phys. Rev. D 35 (1987) 3484.

[4] W.S. Hou, Phys. Lett. B 296 (1992) 179.

[5] M. Luke and M.J. Savage, Phys. Lett. B 307 (1993) 387. 
[6] D. Atwood, L. Reina and A. Soni, SLAC-PUB-95-6927, June 1995.

[7] D. Atwood, L. Reina and A. Soni, SLAC-PUB-95-6962, July 1995.

[8] For a general review of Higgs boson physics, see J.F. Gunion et al., The Higgs Hunter's Guide (Addison-Wesley, Reading, MA, 1990).

191 S.L. Glashow and S. Weinberg, Phys. Rev, D 15 (1977) 1958.

| $10 \mid$ Y.L. Wu and L. Wolfenstein, Phys. Rev. Lett. 73 (1994) 1762 , and references thercin.

[11] B. McWilliams and L.F. Li, Nucl. Phys. B 179 (1981) 62; O. Shanker, ibid. B 206 (1982) 253.
[12] L.J. Hall and S. Weinberg, Phys. Rev. D 48 (1993) R979.

[13] M. Sher and Y. Yuan, Phys. Rev. D 44 (1991) 1461.

[14] D. Chang, W.S. Hou and W.Y. Keung, Phys. Rev, D 48 (1993) 217.

[15] M.S. Alam et al. (CLEO Collaboration), Phys. Rev. Lett. 74 (1995) 2885.

[16] M. Ciuchini et al., Phys. Lett. B 334 (1994) 137.

[17] J.F. Gunion and H.E. Haber, Phys. Rev. D 48 (1993) 5109.

[18] H.F. Ginzburg et al., Nucl. Instrum. Methods 205 (1983) $47 ; 219$ (1984) 5 .

[19] Proceedings of the Second Workshop on Physics Potential and Development of $\mu^{+} \mu^{-}$Colliders, California, November 1994, ed. D.B. Cline (AIP, 1995). 